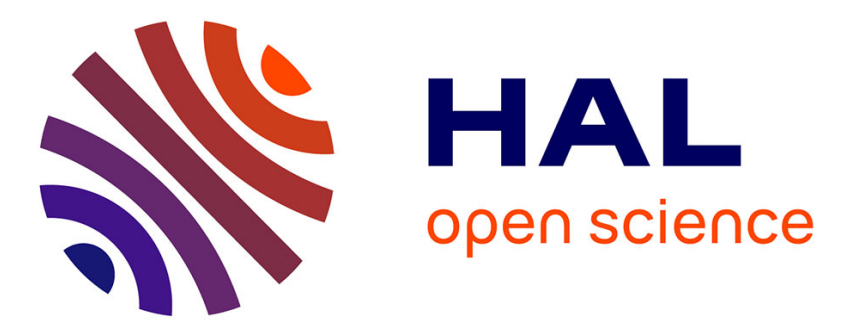

\title{
Les besoins en azote du blé d'hiver jusqu'au début de la montaison
}

Jean-Marc Meynard

\section{To cite this version:}

Jean-Marc Meynard. Les besoins en azote du blé d'hiver jusqu'au début de la montaison. Agronomie, 1985, 5 (7), pp.579-589. hal-00884787

\section{HAL Id: hal-00884787 \\ https://hal.science/hal-00884787}

Submitted on 1 Jan 1985

HAL is a multi-disciplinary open access archive for the deposit and dissemination of scientific research documents, whether they are published or not. The documents may come from teaching and research institutions in France or abroad, or from public or private research centers.
L'archive ouverte pluridisciplinaire HAL, est destinée au dépôt et à la diffusion de documents scientifiques de niveau recherche, publiés ou non, émanant des établissements d'enseignement et de recherche français ou étrangers, des laboratoires publics ou privés. 


\title{
Les besoins en azote du blé d'hiver jusqu'au début de la montaison
}

Jean-Marc MEYNARD

Institut National Agronomique Paris-Grignon, Chaire d'Agronomie, 16, rue Claude-Bernard, F 75231 Paris Cedex 05

Lorsqu'on effectue sur blé, comme la pratique tend à s'en généraliser en France, 2 apports d'engrais azoté en couverture, dont le $2^{\mathrm{e}}$ au début de la montaison (stade " épi $1 \mathrm{~cm}$ "), la dose $\mathrm{Y}$ du $1^{\mathrm{er}}$ apport peut être calculée par un bilan de la forme :

$\mathrm{Y}=$ (besoins du blé jusqu'au stade " épi $1 \mathrm{~cm}$ ») - (fournitures du sol jusqu'à ce stade).

Ce mémoire constitue une contribution à la détermination du jer terme de ce bilan. Nous montrons que la quantité d'azote que peut absorber le blé jusqu'au début de la montaison est limitée supérieurement par une quantité $N$ max, très variable entre peuplements. Sauf si les risques de ne pas contrôler la verse ou le déficit hydrique justifient le choix d'un objectif de rendement peu élevé, les besoins du blé jusqu'au début de la montaison sont égaux à $\mathrm{N}$ max. Il apparaît souhaitable de pouvoir prévoir cette valeur le plus précisément possible en sortie d'hiver, car un surplus d'engrais par rapport aux besoins risquerait d'être rendu indisponible par lessivage ou réorganisation.

Nous proposons un modèle d'estimation de $\mathrm{N}$ max en fonction de la densité de plantes en sortie d'hiver ; les paramètres du modèle dépendent de la date de semis du blé, de la variété et du pédoclimat.

Les résultats nous amènent à discuter en conclusion l'incidence de l'avancement, par rapport à la pratique d'il y a 10 ou 15 ans, de la date du $2^{e}$ apport d'azote sur le rôle respectif des 2 apports, et la modulation de leur importance selon l'état du peuplement en fin d'hiver.

Mots clés additionnels :Fertilisation azotée, fractionnement de la fumure, objectif de rendement, composantes du rendement, densité de plantes, modèle de croissance potentielle, teneur en azote des parties aériennes, stade « épi l $\mathrm{cm} »$.

\begin{abstract}
Nitrogen requirements of winter wheat until the beginning of stem elongation.
It is becoming usual in France to make two nitrogen applications as top dressings on winter wheat, the second application being made at the very beginning of stem elongation (stem + apex length $=10 \mathrm{~mm}$ ). The amount (Y) of the first supply can be calculated from the following equation : $Y=$ (Nitrogen requirements of the crop till « $10 \mathrm{~mm}$ stage $")$ - (Nitrogen supplied by the soil). This paper provides elements for determining the first term of this equation. An upper limit was shown for the amount of nitrogen which can be found in the plant at the beginning of stem elongation $(\mathrm{N}$ max). The nitrogen requirements of a wheat crop till this stage were found to equal $\mathrm{N}$ max, unless there were major risks of lodging or water deficiency and consequently the target yield was low. It would thus be useful to predict $N$ max as early as possible at the end of winter, since any excess of nitrogen fertilizer may become unavailable for later stages, because of leaching or reorganization. A model for early evaluation of $\mathrm{N}$ max is proposed, depending on the number of plants per unit area ; the parameters of the model are related to sowing date, variety and pedoclimate. In conclusion, we examine the consequences of the date of the second nitrogen application on the respective roles of the two applications, and how the two should be adjusted relative to each other according to the actual state of the crop at the end of winter.
\end{abstract}

Additional key words : Nitrogen fertilization, nitrogen splitting, yield target, yield components, plant density, potential growth model, plant nitrogen content.

\section{INTRODUCTION}

Considérant que le rôle de la fumure azotée est de compléter la fourniture du sol pour satisfaire les besoins de la culture, HÉBERT (1969) a proposé de déterminer la dose totale d'engrais azoté à appliquer sur blé par un bilan prévisionnel de la forme :

Dose d'engrais $=$ (besoins du blé) - (fourniture du sol) 
L'intérêt pratique d'une telle équation repose sur la possibilité de prédire le plus précisément ses différents termes (RÉMY, 1981) : les besoins sur l'ensemble du cycle sont estimés à $3 \mathrm{~kg} / \mathrm{q}$ de rendement attendu ; les fournitures du sol peuvent être évaluées de manière relativement satisfaisante à l'aide de références, telles celles vulgarisées par l'ITCF (1978). Après une phase de tests à large échelle et de mise au point (VIAUX, 1980 ; MEYNARD et al., 1981), ce bilan prévisionnel est maintenant opérationnel et largement diffusé.

RÉMY \& HÉBERT (1977) ont proposé d'utiliser un bilan analogue pour raisonner le fractionnement de la fumure. Lorsqu'on effectue, comme la pratique tend à s'en généraliser en France, un $1^{\text {er }}$ apport d'engrais azoté en " sortie d'hiver » (apport « tallage ", début à fin février selon les régions) et un $2^{\mathrm{e}}$ au début du redressement (apport «montaison » au stade épi $1 \mathrm{~cm}$ ), on peut effectivement envisager de calculer la dose du $1^{\text {er }}$ apport par la relation suivante :

$\left(\begin{array}{c}\text { dose } \\ \text { ler }^{\text {apport }}\end{array}\right)=\left(\begin{array}{c}\text { besoins du blé jusqu'au } \\ \text { stade épi } 1 \mathrm{~cm}\end{array}\right)-\left(\begin{array}{c}\text { fournitures du sol } \\ \text { avant ce stade }\end{array}\right)(2)$

Mais la mise en œuvre de ce bilan prévisionnel butte sur le manque de références concernant, en particulier, les besoins précoces de la culture (VIAUX, 1980). Ce mémoire constitue une contribution à la mise au point de telles références.

On s'attachera :

1 - à préciser la notion de «besoins précoces". D'après la définition de COÏC (1956), ils correspondent à la "quantité d'azote que le blé doit absorbèr (avant le début de la montaison) pour obtenir le rendement optimal $»$. On étudiera donc les relations existant entre azote absorbé au stade épi $1 \mathrm{~cm}$ et élaboration du rendement. Ces relations sont susceptibles d'être sensiblement différentes selon la quantité totale d'azote à la disposition du blé sur l'ensemble du cycle (CoÎ́C, 1949), c'est-à-dire, si on calcule la dose totale d'engrais à l'aide d'un bilan prévisionnel, selon l'objectif de rendement ;

2 - à proposer, à la suite de cette analyse, un modèle de prévision des besoins précoces.

\section{MATÉRIEL ET MÉTHODES}

\section{A. Dispositif expérimental}

Les variations de l'absorption précoce d'azote ont été étudiées et modélisées à partir des résultats de 111 essais mettant en comparaison au moins 2 doses d'apport "tallage " et, dans 70 cas, présentant un témoin non fertilisé. Dans ces essais, 2 caractéristiques susceptibles d'influer sur la croissance précoce et sur l'absorption d'azote varient fortement : la densité de plantes $\left(50\right.$ à $\left.580 / \mathrm{m}^{2}\right)$ et la date de semis $(9 / 10$ au 16/02).

L'interaction entre absorption précoce d'azote et nutrition sur l'ensemble du cycle a été étudiée à partir de 26 de ces essais (dits complets), qui combinaient, avec les différentes doses "tallage ", 2 à 4 variantes de dose totale. Ces dernières ont été calculées par des bilans prévisionnels, les objectifs de rendement étant adaptés sur chaque essai à l'état de la culture en fin d'hiver (MEYNARD, 1983). Les 2 apports d'engrais ont été effectués en couverture, le $1^{\text {er }}$ pendant la $2^{\mathrm{e}}$ quinzaine de février, le $2^{\mathrm{e}}$ au stade " épi $1 \mathrm{~cm}$ ». Tous les traitements expérimentaux ont été répétés sur 2 ou 3 blocs randomisés.

De manière à vérifier la généralité des résultats obtenus, le dispositif a été répété plusieurs années successives, sur différentes variétés, dans 2 petites régions du Bassin Parisien : en Champagne crayeuse (sols de rendzines développés sur craie), pendant 4 années, sur la variété «Talent » et dans le Noyonnais (sols lessivés développés sur limons loessiques), pendant 3 années, sur les variétés «Fidel» et «Lutin» (exceptionnellement «Arminda »).

Les principales caractéristiques du dispositif sont données par le tableau 1. Les annexes 1 et 2 présentent les grands traits du milieu des divers essais. Les différentes années ont permis d'explorer une partie importante de la gamme de variation des régimes thermiques d'hiver et de début de printemps : hiver doux en 1979-80 et 1982-83, hiver très rigoureux avec gel important du blé dans certains essais en 1978-79; début de printemps froid en 1978 et 1982, chaud en 1981. La répartition des pluies au printemps a, dans tous les cas, été favorable à une solubilisation rapide des 2 apports d'engrais.

\section{B. Contrôles expérimentaux}

\section{Absorption d'azote jusqu'au stade "épi $1 \mathrm{~cm}$ "}

Un prélèvement de plantes a été réalisé au début de la montaison, sur 10 placettes $(2$ lignes $\times 50 \mathrm{~cm})$ par traitement, pour détermination du nombre de plantes à l'unité de surface, de la biomasse et de la teneur en azote de leurs parties aériennes (analyses d'azote: méthode KJELDAHL, après réduction des nitrates). La quantité totale d'azote absorbé à ce stade par le peuplement a été estimée en retenant l'hypothèse d'une proportionnalité entre quantités d'azote contenues dans les parties aériennes et dans les racines (tabl. 2).

Le stade "épi $1 \mathrm{~cm}$ " correspond au moment où, sur 50 p. 100 des brins-maîtres, la longueur " tige + épi » (distance entre la base du plateau de tallage et le sommet de l'apex) est supérieure ou égale à $10 \mathrm{~mm}$. Ce stade correspond sensiblement, pour les variétés « Lutin » et "Talent », au stade B II de l'apex (échelle de JONARD); pour "Fidel » et " Arminda », il lui est, de quelques jours, antérieur. Le stade effectif de chaque peuplement au moment du prélèvement a été déterminé par observation à la loupe binoculaire de 20 à 30 brins-maîtres par essai : les longueurs «tige + épi » médianes observées se situent dans une fourchette de $8-18 \mathrm{~mm}$; dans cette gamme, la biomasse aérienne s'accroît proportionnellement à la longueur " tige + épi » (MASLE, comm. pers.). Les traitements expérimentaux ont donc tous pu être comparés au stade épi $1 \mathrm{~cm}$, soit directement sur la biomasse mesurée, soit sur la biomasse obtenue par la correction suivante :

Biomasse au stade " épi $1 \mathrm{~cm}$ " = Biomasse mesurée $\times \frac{1 \mathrm{~cm}}{\text { longueur « tige }+ \text { épi » mesurée }(\mathrm{cm})}$ 
TABLEAU 1

Caractéristiques des essais.

Characteristics of the experimental plots.

\begin{tabular}{|c|c|c|c|c|c|c|c|c|c|c|}
\hline \multirow[b]{2}{*}{ 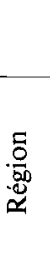 } & \multicolumn{4}{|c|}{$\begin{array}{c}\text { Ensemble des } \\
\text { essais }\end{array}$} & \multicolumn{5}{|c|}{ Essais complets } & \multirow[b]{2}{*}{$\begin{array}{c}\text { Doses totales } \\
\text { d'azote }(\mathrm{N} \mathrm{kg} / \mathrm{ha}) \\
\text { croisées avec } \\
\text { les doses } \\
\text { du premier apport }\end{array}$} \\
\hline & 怤 & $\begin{array}{l}\text { D̆ } \\
\text { E } \\
\text { Z }\end{array}$ & : & $\begin{array}{l}\mathbb{8} \cdot \overline{7} \\
\dot{0}: 0\end{array}$ & $\begin{array}{l}\text { Précédent } \\
\text { cultural }\end{array}$ & 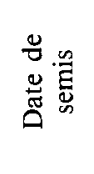 & $\begin{array}{l}\text { Densité } \\
\text { de pieds } \\
\text { sortie } \\
\text { d'hiver }\end{array}$ & $\begin{array}{l}\text { Doses du } \\
1^{\text {er }} \text { apport } \\
\text { comparées } \\
(\mathrm{N} \mathrm{kg} / \mathrm{ha})\end{array}$ & $\begin{array}{l}\text { Objectifs de } \\
\text { rendement } \\
(\mathrm{q} / \mathrm{ha})\end{array}$ & \\
\hline \multirow{18}{*}{ 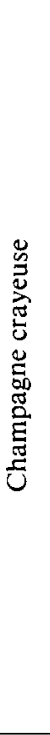 } & 1977 & 8 & Talent & EER & $\begin{array}{l}\text { Betterave } \\
\text { sucrière }\end{array}$ & $15 / 11$ & 270 & $2060 \quad 100$ & 577083 & 120160200 \\
\hline & & & & $\begin{array}{l}\text { EEB } \\
\text { EON }\end{array}$ & $\begin{array}{l}\ldots \\
\ldots\end{array}$ & $\begin{array}{l}15 / 11 \\
22 / 11\end{array}$ & $\begin{array}{l}250 \\
250\end{array}$ & $\begin{array}{lll}20 & 60 & 100 \\
20 & 60 & 100\end{array}$ & $\begin{array}{lll}57 & 70 & 83 \\
57 & 70 & 83\end{array}$ & $\begin{array}{lll}120 & 160 & 200 \\
120 & 160 & 200\end{array}$ \\
\hline & 1978 & 9 & Talent & ED2 & $\ldots$ & $28 / 10$ & 185 & 3060 & $\begin{array}{llll}57 & 70 & 83 & 97\end{array}$ & $\begin{array}{llll}130 & 170 & 210 & 250\end{array}$ \\
\hline & & & & EN1 & $\cdots$ & $23 / 11$ & 325 & $2060 \quad 100$ & $57 \quad 7083$ & 130170210 \\
\hline & & & & EN2 & $\ldots$ & $28 / 11$ & 338 & $2060 \quad 100$ & $57 \quad 7083$ & $\begin{array}{lll}130 & 170 & 210\end{array}$ \\
\hline & & & & EN31 & $\ldots$ & $29 / 11$ & 120 & $\begin{array}{llll}40 & 80 & 120\end{array}$ & 5770 & 130170 \\
\hline & & & & EN32 & $\ldots$ & $29 / 11$ & 290 & $4080 \quad 120$ & 5770 & $130 \quad 170$ \\
\hline & & & & EN33 & $\cdots$ & $29 / 11$ & 440 & $\begin{array}{lll}40 & 80 & 120\end{array}$ & 5770 & $120 \quad 170$ \\
\hline & 1979 & 15 & Talent & ES21 & $\ldots$ & $20 / 10$ & 54 & 2040 & 3050 & $45 \quad 105$ \\
\hline & & & & ED50 & $\ldots$ & $21 / 10$ & 92 & 1540 & 3555 & 50110 \\
\hline & & & & EN5 & $\ldots$ & $21 / 11$ & 256 & 204060 & $\begin{array}{lll}53 & 66 & 79\end{array}$ & $\begin{array}{llll}100 & 140 & 180\end{array}$ \\
\hline & & & & ES1K & $\ldots$ & $12 / 12$ & 165 & 2040 & 7090 & 165225 \\
\hline & & & & ES2M & $\ldots$ & $12 / 12$ & 372 & 4060 & 7090 & 165225 \\
\hline & & & & ES1I & $\ldots$ & $15 / 02$ & 370 & 2040 & 5676 & 12080 \\
\hline & & & & ED6A & $\ldots$ & $16 / 02$ & 263 & 204060 & 435676 & $\begin{array}{llll}80 & 120 & 180\end{array}$ \\
\hline & & & & ED5A & $\ldots$ & $16 / 02$ & 278 & 2040 & 435676 & $\begin{array}{lll}80 & 120 & 180\end{array}$ \\
\hline & & & & EN6 & $\cdots$ & $17 / 02$ & 450 & 204060 & $43 \quad 5669$ & 80120160 \\
\hline & 1980 & 4 & Talent & - & - & - & - & - & - & - \\
\hline \multirow{10}{*}{ 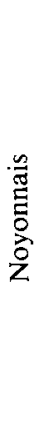 } & 1981 & 12 & Fidel & $\mathrm{AZZ}$ & $\cdots$ & $22 / 11$ & 283 & 4090 & 6580 & 110160 \\
\hline & & $\& 13$ & Lutin & - & - & - & - & - & - & - \\
\hline & 1982 & & & $\int$ FA2 & $\ldots$ & $16 / 11$ & 317 & 4070 & 6580 & $135 \quad 180$ \\
\hline & & 11 & Fidel & I5B & $\ldots$ & $20 / 11$ & 212 & 4070 & 6580 & $125 \quad 170$ \\
\hline & & & & $15 \mathrm{~A}$ & $\ldots$ & $20 / 11$ & 319 & 4070 & 6580 & $125 \quad 170$ \\
\hline & & 9 & Lutin & $\{$ FA5 & $\ldots$ & $17 / 11$ & 236 & 4070 & 6580 & $135 \quad 180$ \\
\hline & & 3 & & FA6 & & $17 / 11$ & 275 & 4070 & 6580 & $135 \quad 180$ \\
\hline & & & & $\{$ FA9 & Haricot & $7 / 10$ & 281 & 4070 & 6580 & $125 \quad 170$ \\
\hline & & 2 & Arminda & $\{$ FAl & $\begin{array}{l}\text { Pomme } \\
\text { de terre }\end{array}$ & $13 / 10$ & 202 & 4070 & 6075 & 120165 \\
\hline & 1983 & $\begin{array}{r}18 \\
\& 10\end{array}$ & $\begin{array}{l}\text { Fidel } \\
\text { Lutin }\end{array}$ & - & - & - & - & - & - & - \\
\hline
\end{tabular}

\section{Modalités d'élaboration $d u$ rendement}

Les contrôles précédents ont été complétés par l'observation des séquences de tallage, dont MASLEMEYNARD $(1981 b ; 1985)$ a montré qu'elles constituaient un bon révélateur de la chronologie d'installation d'une compétition dans le peuplement. L'observation a été faite au début de la montaison, sur 60 à 100 plantes par traitement expérimental, pour 3 à 4 essais chaque année en $1977,78,79$ et pour la quasitotalité des essais en 1982 et 83 .

Pour les phases ultérieures, on a privilégié les composantes du rendement nombre d'épis $/ \mathrm{m}^{2}$ et nombre de grains $/ \mathrm{m}^{2}$. Le poids de 1000 grains est, dans nos conditions (absence d'apport tardif), peu influencé par la fumure azotée et dépend surtout de la nutrition hydrique et carbonée après floraison. Le nombre de grains $/ \mathrm{m}^{2}$ peut être considéré, de ce fait (BoIFFIN et al., 1981), comme le meilleur critère pour une appréciation globale de l'influence de la fumure azotée sur l'élaboration du rendement. Les nombres d'épis et de grains $/ \mathrm{m}^{2}$ ont été déterminés à partir d'un prélèvement à maturité de 10 placettes par traitement expérimental.

\section{Etat du milieu}

On a effectué différentes observations et mesures sur le milieu dans le but d'identifier l'intervention éventuelle d'autres facteurs limitants que l'azote : analyses chimiques, profils culturaux, notation des symptômes de maladies, de la présence des ravageurs et d'adventices, de verse... Ces observations nous permettent d'exclure l'existence, pour les différents essais étudiés, d'un effet dépressif conséquent des maladies, parasites, adventices, ou carences en $P$ ou $K$ sur la croissance anté-floraison et le nombre de grains $/ \mathrm{m}^{2}$ (MEYNARD et al., 1982 - Champagne crayeuse ; MEYNARD \& SEBILLOTTE, 1982 - Noyonnais). 
TABLEAU 2

Modalités d'estimation du rapport entre les quantités d'azote contenues dans les racines et dans les parties aériennes au début du redressement (blé d'hiver fertilisé, au champ).

Literature basis for the calculation of the ratio between the amounts of nitrogen in the roots and in the aerial parts at the beginning of stem elongation (fertilizer plots of winter wheat, in the field).

\begin{tabular}{|c|c|c|c|c|}
\hline \multirow{2}{*}{$\begin{array}{c}\text { Rapport } \\
\text { Biomasse racinaire } \\
\text { Biomasse aérienne }\end{array}$} & \multicolumn{2}{|c|}{ Teneurs en azote des parties } & \multicolumn{2}{|c|}{ D'où rapport } \\
\hline & Aériennes & Racinaires & $\begin{array}{l}\text { des teneurs } \\
\text { en azote }\end{array}$ & $\begin{array}{c}\text { des quantités } \\
\text { d'azote }\end{array}$ \\
\hline$\simeq 1,0\left(^{*}\right)$ & 3,5 à $5,5\left({ }^{* *}\right)$ & 1,0 à $1,7(* * *)$ & $\simeq 0,3$ & $\simeq 0,3$ \\
\hline
\end{tabular}

$\left({ }^{*}\right)$ D'après BALDY, 1973.

(**) D'après Jonard \& Odent, 1967 ; Puia et al., 1978 ; Karlen \& Whitney, 1980 ; Masle, 1980 ; Saut, 1981.

(***) D'après Chatilov \& SAFONOv, 1970 ; Puia et al., 1978.

\section{RÉSULTATS}

\section{A. Relations entre azote absorbé au début de la mon- taison et élaboration du rendement}

\section{Influence de doses croissantes d'engrais sur l'absorption précoce d'azote}

Dans 64 essais sur les 70 présentant un témoin non fertilisé, la biomasse aérienne au stade épi $1 \mathrm{~cm}$ est augmentée de manière significative (seuil de probabilité $5 \mathrm{p}$. 100) par les premières doses testées; dans nos milieux, la fourniture d'azote par le sol n'est donc pas, en général, suffisante pour satisfaire les besoins précoces du blé ; on retrouve là des résultats classiques (par exemple Col̈c, 1956 ; SPIEA, 1968).

Dans un peu moins de la moitié des essais (46 sur 111), la biomasse aérienne présente un plateau pour les plus fortes doses d'engrais appliquées. Le niveau de ce plateau est extrêmement variable : de moins de $10 \mathrm{~g} / \mathrm{m}^{2}$ (essais présentant moins de 100 plantes $/ \mathrm{m}^{2}$ à la suite du gel de l'hiver 1978-79) à plus de $100 \mathrm{~g} / \mathrm{m}^{2}$ (essais semés en octobre à densité élevée). Dans les 65 autres essais, la gamme de doses d'engrais explorée n'a pas été suffisante pour que ce plateau soit observé.

La teneur en azote des organes aériens varie de 2 à environ 4 p. 100 sur les traitements pour lesquels l'azote a été facteur limitant de la croissance avant le début de la montaison (partie ascendante des courbes de réponse de la biomasse aérienne à la fumure azotée, fig. 1a) ; dans chaque essai, elles augmentent avec la dose d'engrais. Lorsque l'azote n'a plus d'effet sur la croissance, la teneur est comprise entre 3,2 et 5,5 p. 100 (90 p. 100 entre 3,7 et 5,3 p. 100, fig. 1 b). Cette gamme est peu différente de celle observée par d'autres auteurs sur des essais largement fertilisés (KARLEN \& WHITNEY, 1978 ; MASLE-MEYNARD, 1980 ; SAUT, 1981). A l'exception de quelques essais, sur lesquels les teneurs les plus faibles $(3,2$ à 3,8 p. 100) ont été observées, la teneur en azote augmente peu sur le plateau des courbes (moins de 0,3 p. 100) : on n'observe pratiquement pas de consommation de luxe.

La quantité d'azote que peut absorber un peuplement de blé dans une situation donnée apparaît donc bornée supérieurement à un niveau très variable dépendant de l'état de croissance du peuplement ( 2 à $80 \mathrm{~kg} \mathrm{~N} / \mathrm{ha}$, racines comprises, pour nos essais).

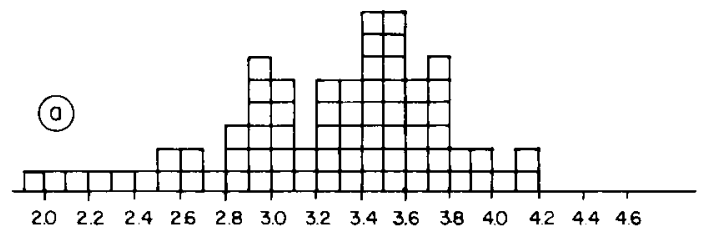

(b)

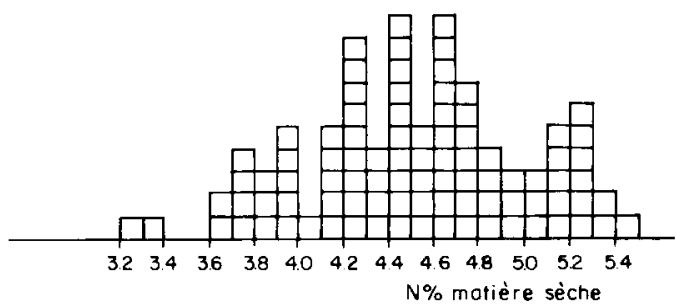

Figure 1

Teneur en azote des parties aériennes du blé au stade «Epi $1 \mathrm{~cm} »$, selon que cet élément est, ou non, facteur limitant de la croissance. Figure 1a

Si l'azote est facteur limitant de la croissance.

Figure $1 \mathrm{~b}$

Si l'azote n'est pas facteur limitant de la croissance.

$\square$ I traitement experimental.

Dans chaque classe, la borne supérieure est exclue. Seuls ont été reportés sur chacun des histogrammes les traitements expérimentaux pour lesquels les courbes de réponse à la fumure azotée permettaient de déterminer, de manière certaine, si l'azote a, ou non, été facteur limitant de la croissance.

Nitrogen content of the aerial parts at the beginning of stem elongation when:

a) nitrogen is a limiting factor,

b) nitrogen is not limiting.

Du point de vue de l'évaluation des besoins précoces, cette observation amène 2 questions :

- Les besoins de la culture sont-ils égaux à cette quantité maximale absorbable ou lui sont-ils parfois inférieurs? Autrement dit, le nombre de grains $/ \mathrm{m}^{2}$ est-il affecté par une augmentation de biomasse aérienne lorsque celle-ci est créée par une augmentation de la dose du $1^{\text {er }}$ apport d'azote ?

- Est-il préjudiciable d'apporter au blé une quantité d'azote supérieure à celle qu'il peut absorber jusqu'au début de la montaison ? On peut, en effet, penser que l'azote du $1^{\text {er }}$ apport qui subsiste dans le sol au stade «épi $1 \mathrm{~cm}$ " risque d'être lessivé - en particulier sur sol filtrant ou superficiel - ou réorganisé et, de ce fait, seulement partiellement utilisable pendant la suite du cycle de la culture. 
2. Relation entre la croissance précoce et le nombre de grains $/ \mathrm{m}^{2}$, à différents niveaux de fumure azotée

Le tableau 3 donne les éléments d'une réponse aux 2 questions qui viennent d'être posées; il confronte les effets d'une augmentation de la dose tallage sur :

- d'une part, la biomasse aérienne au stade « épi $1 \mathrm{~cm}$,

- d'autre part, en interaction avec la dose totale d'engrais, le nombre de grains $/ \mathrm{m}^{2}$ des mêmes essais.

Dans les tests mis en œuvre, il est important, pour interpréter ces données, que le risque de $1^{\text {re }}$ espèce soit aussi faible que possible et, simultanément, la puissance aussi élevée que possible. Etant donné la taille de nos échantillons, nous avons adopté un risque de $1^{\text {re }}$ espèce de $10 \mathrm{p} .100$ dans les tests de comparaison de moyennes sur la biomasse aérienne, et de 5 p. 100 dans l'analyse de variance sur le nombre de grains $/ \mathrm{m}^{2}$. On s'assure ainsi une puissance de 0,9 pour la mise en évidence de différences de l'ordre de 1,2 à 1,3 fois l'écart-type sur les 2 variables.

a) Cas des essais où la biomasse aérienne au stade " épi $1 \mathrm{~cm}$ " ne répond pas à un supplément d'apport au tallage

Dans la majorité des essais, le nombre de grains n'est pas influencé par la répartition de l'engrais entre $1^{\text {er }}$ et $2^{\mathrm{e}}$ apport, quelle que soit la dose totale appliquée. L'engrais apporté en trop en sortie d'hiver a donc eu la même efficacité pendant la montaison que l'azote apporté au début de cette phase.
Sur 3 essais, au contraire, on observe un effet négatif des apports «tallage" élevés, de l'ordre de 10 p. 100 , sur les nombres d'épis et de grains $/ \mathrm{m}^{2}$. L'origine de ce comportement est difficile à déterminer avec certitude ; on remarque cependant qu'il est observé en 1978 seulement, année à printemps froid et pluvieux, favorable au lessivage et à la réorganisation de l'engrais et qu'il concerne des essais pour lesquels l'excédent de fumure «tallage» par rapport à l'absorption du blé est très important (supérieur à $40 \mathrm{~kg} \mathrm{~N} / \mathrm{ha}$ ).

Il existe ainsi un risque qu'un apport précoce excédentaire puisse conduire à une moindre efficience de l'engrais. Il apparaît donc souhaitable de ne pas fournir au blé, en sortie d'hiver, une quantité d'azote trop supérieure à celle qu'il pourra absorber jusqu'au stade " épi $1 \mathrm{~cm}$ ". VIAUX (1983) aboutit à une conclusion similaire en fondant son argumentation sur la faible valeur du coefficient d'utilisation dv $1^{\text {er }}$ apport d'azote (RÉMY \& VIAUX, 1980 - mesure effectuée sur l'ensemble du cycle à partir de l'application d'engrais marqué par ${ }^{15} \mathrm{~N}$ ).

\section{b) Cas des essais où la biomasse aérienne au stade " épi $1 \mathrm{~cm}$ " répond à la dose " tallage"}

7 essais «complets » correspondent seulement à ce cas de figure, sans doute en raison de la gamme des doses «tallage » appliquées : jamais de $1^{\text {er }}$ apport nul dans les comparaisons à dose totale constante.

Pour 4 d'entre eux, l'effet d'une augmentation de l'apport «tallage " sur le nombre de grains $/ \mathrm{m}^{2}$ est négatif pour les doses totales les plus faibles; il est

TABLEAU 3

Effet d'une augmentation de la dose «tallage » sur le nombre de grains $/ \mathrm{m}^{2}$. Interaction avec la dose totale d'engrais azoté. Effect of the first nitrogen fertilizer application on grain number. Interaction with total fertilizer rate.

1 symbole $=1$ essai complet du tableau 2

- Champagne 1977

- Champagne 1978

A Champagne 1979

$\square \quad$ Noyonnais 1981

O Noyonnais 1982

Effet d'une augmentation de la dose « tallage » sur la biomasse aérienne au stade " épi $1 \mathrm{~cm}$ "

Effet d'une augmentation de la dose « tallage » sur le nombre de grains $/ \mathrm{m}^{2}$

Identique quelle que soit la dose totale testée (interaction dose « tallage »-dose totale non significative au seuil de probabilité $5 \%$ )
Diminution du nombre de grains $/ \mathrm{m}^{2}$ significative au seuil de probabilité $5 \%$

Pas de variation du nombre de grains $/ \mathrm{m}^{2}$ significative au seuil de probabilité $5 \%$ $\bullet$

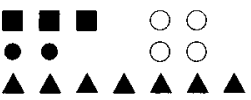

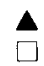

Augmentation du nombre de grains $/ \mathrm{m}^{2}$ significative au seuil de probabilité $5 \%$

Différent selon la dose Diminution du nombre de totale (interaction dose grains $/ \mathrm{m}^{2}$ pour les doses « tallage . »-dose totale totales faibles significative au seuil de probabilité $5 \%$ )

Diminution pour les doses totales fortes

\begin{tabular}{ll}
\hline $\begin{array}{l}\text { Pas de variation pour les } \\
\text { doses totales fortes }\end{array}$ & 0 \\
\hline $\begin{array}{l}\text { Augmentation pour les doses } \\
\text { totales fortes }\end{array}$ & $\bullet$
\end{tabular}


positif ou nul pour les plus fortes. Pour les 3 autres, l'interaction dose totale-dose "tallage " n'est pas significative au seuil de probabilité retenu (5 p. 100), mais les sens de variation du nombre de grains $/ \mathrm{m}^{2}$ sont similaires. Pour les 7 essais, les variations des nombres d'épis et de grains $/ \mathrm{m}^{2}$ sont parallèles.

L'augmentation du potentiel de production du peuplement, liée à l'augmentation de biomasse aérienne au stade « épi $1 \mathrm{~cm}$ » (MASLE, 1980), n'est donc pas valorisée lorsque la dose totale d'azote est faible. On crée alors, selon le schéma décrit par CoḮ (1956), un besoin supplémentaire d'azote pendant la montaison, qui ne peut être satisfait par une dose "montaison » d'autant plus faible que la dose "tallage » est plus élevée. Cependant, la combinaison dose "tallage » élevée-dose totale élevée n'a un effet positif sur le rendement que si ces doses d'engrais importantes ne favorisent pas l'expression d'un facteur limitant du poids de 1000 grains, tel que la verse, les maladies cryptogamiques ou le déficit hydrique. Ainsi, sur l'un die nos essais (ED 2,1978) où la dose totale appliquée a été de $250 \mathrm{~kg} /$ ha pour un objectif de rendement de $83 \mathrm{q} / \mathrm{ha}$, le peuplement ayant obtenu le plus fort nombre de grains $/ \mathrm{m}^{2}$ (dose «tallage » $60 \mathrm{~kg} / \mathrm{ha}$ ) a versé au stade laiteux du grain, ce qui a entraîné une chute de son poids de 1000 grains d'environ 10 p. 100 . Le meilleur rendement de l'essai a finalement été observé sur le traitement : dose «tallage » $30 \mathrm{~kg} / \mathrm{ha}$.

On a donc intérêt, pour obtenir un rendement élevé, à rechercher la biomasse aérienne maximale au stade " épi $1 \mathrm{~cm}$ ", mais à condition que l'objectif de rendement soit adapté au potentiel de la culture et que la protection contre la verse et les maladies soit suffisante. Inversement, si les risques de ne pas contrôler ces facteurs limitants et/ou le déficit hydrique justifient le choix d'un objectif de rendement peu élevé, une carence précoce en azote (qui entraîne une diminution du potentiel de nombre de grains $/ \mathrm{m}^{2}$ ), apparaît souhaitable.

\section{B. Eléments pour la prévision des besoins précoces}

Le schéma d'interprétation de l'influence du $1^{\text {er }}$ apport d'engrais sur l'élaboration du rendement met en évidence le rôle clé que doit avoir, dans l'évaluation des besoins précoces, le niveau maximum de biomasse aérienne qu'il sera possible d'atteindre au stade « épi $1 \mathrm{~cm}$ » (BA 1).

La quantité d'azote nécessaire au blé pour atteindre ce niveau de biomasse est :

$$
\mathrm{N} \max =\mathrm{BA} 1 \times \tau \times(1+\rho)
$$

où $\tau$ est la teneur en azote total des parties aériennes du blé au début de la montaison, lorsque la nutrition azotée n'est pas limitante $(\tau \simeq 4,5 \pm 0,8 \mathrm{p}, 100)$, $\rho$ est le rapport entre les quantités d'azote contenues dans les racines et dans les parties aériennes $(\rho \simeq 0,3$, d'après les données du tableau 2).

Si la quantité d'azote à la disposition de la culture est inférieure à $\mathrm{N}$ max, le potentiel de nombre de grains $/ \mathrm{m}^{2}$ en sera diminué ; si elle est supérieure à $\mathrm{N}$ max, on risquera une mauvaise efficience de l'engrais azoté.

Les besoins précoces du peuplement, pour l'obtention d'un rendement élevé avec une efficience maxi- male de l'azote, sont donc égaux à $\mathrm{N}$ max et leur estimation est subordonnée à la prévision du terme le plus variable de la relation (3) : BA 1.

\section{Modèle retenu pour la prévision de la biomasse aérienne début montaison}

Dans une perspective de mise au point de références pour la conduite de la culture, le modèle recherché doit :

- prendre en compte les caractéristiques du peuplement, du milieu et du système de culture dont les variations entre parcelles d'agriculteurs sont suffisamment importantes pour influer de manière sensible sur BA 1 : en particulier la date de semis, la densité de plantes, la température subie par le peuplement, la variété...,

- ne nécessiter que des données d'accès aisé.

Face à cette $2^{\mathrm{e}}$ exigence, les modèles de croissance mécanistes, à pas de temps court, basés en général sur le bilan photosynthétique journalier (par exemple : DE WIT et al., 1971 ; HODGES \& KANEMASU, 1977) apparaissent mal adaptés : ils mettent en œuvre de nombreux paramètres dont les modalités de variation sont complexes et encore insuffisamment explicitées ; bien qu'ils soient relativement fiables pour la simulation de croissances journalières, leur qualité prédictive à échéance de plusieurs mois est actuellement, de ce fait, souvent insuffisante (RICKMAN et al., 1975 ; VAN KeUlen \& DE Milliano, 1984).

Nous nous sommes donc tourné vers un modèle beaucoup plus simple, celui de SHINOZAKI \& KIRA (cités par WILLEY \& HEATH, 1969), qui exprime la réponse de la biomasse aérienne à la densité de plantes (NP), en utilisant un petit nombre de paramètres ayant une signification biologique :

$$
\frac{N P}{B A 1}=a+b N P \text {. }
$$

Ce modèle traduit bien, d'après DONALD (1963), la diminution du poids sec d'une plante liée à l'augmentation de la compétition pour la lumière lorsque la densité augmente. Les paramètres «a " (inverse du poids sec d'une plante en l'absence de compétition) et " b " (inverse de la biomasse aérienne par unité de surface quand la densité de plantes tend vers l'infini) dépendent tous deux, mais selon des lois différentes:

- de la somme de températures levée-stade "épi $1 \mathrm{~cm} »$, très variable entre peuplements, selon leur variété, leur date de semis et les microclimats auxquels ils sont soumis (JONARD, 1964) ;

- de la variété, en liaison avec son port, la forme de ses feuilles...

On peut, d'après Willey \& HEATH (1969), proposer les lois de variation suivantes:

$$
\begin{aligned}
& \mathrm{a}=\mathrm{W}_{0} \mathrm{e}^{-\lambda(\Sigma \boldsymbol{\theta})} \\
& \mathrm{b}=\mathrm{K}\left(1-\mathrm{e}^{-\lambda(\Sigma \boldsymbol{\theta})}\right)
\end{aligned}
$$

$\Sigma \theta$ : somme de températures levée-stade « épi $1 \mathrm{~cm}$ » $\mathrm{W}_{0}, \lambda, \mathrm{K}$ : constantes variétales.

Cependant, le modèle (4) n'est pas satisfaisant dans la gamme des faibles densités où la compétition pour 
la lumière peut ne pas avoir encore commencé au début de la montaison (MASLE, 1985). Dans ces conditions, le poids sec d'une plante est indépendant du nombre de plantes qui l'entourent :

$$
\frac{\mathrm{BA} 1}{\mathrm{NP}}=\alpha
$$

L'installation d'une compétition pour la lumière entre plantes avant le stade « épi $1 \mathrm{~cm}$ » dépend largement de l'indice foliaire du peuplement et, secondairement, du port des feuilles (DONALD, 1963). L'existence d'une très forte corrélation, aux stades jeunes de la culture, entre surface foliaire et biomasse aérienne (AASE, 1978 ; MASLE, 1985), autorise à exprimer le domaine de validité des relations (4) et (7) en référence à une biomasse aérienne seuil (BA Is). A priori, BA $1 \mathrm{~s}$ est susceptible de ne pas être identique entre variétés de port différent.

D'où l'écriture suivante du modèle :

- si $\alpha \mathrm{NP} \leqslant \mathrm{BA} 1 \mathrm{~s}, \mathrm{BA} 1=\alpha \mathrm{NP}$

- $\operatorname{si} \alpha \mathrm{NP} \geqslant \mathrm{BA} 1 \mathrm{~s}, \mathrm{BA} 1=\frac{\mathrm{NP}}{\mathrm{a}+\mathrm{b} \mathrm{NP}}$

avec $\mathrm{BA} 1=\mathrm{BA} 1 \mathrm{~s}$ quand $\mathrm{NP}=\frac{\mathrm{BA} 1 \mathrm{~s}}{\alpha}$

soit :

$$
\alpha=\frac{1-\mathrm{b} \cdot \mathrm{BA} \text { is }}{\mathrm{a}}
$$

Dans les paragraphes suivants, nous allons déterminer BA $1 s$ et étudier l'ajustement du modèle à nos données expérimentales. La validation des relations (5) et (6) ne sera cependant pas abordée ici, faute de données multilocales.

\section{Détermination de la biomasse aérienne seuil (BA Is)}

Les traitements expérimentaux retenus sont ceux correspondant au plateau des courbes de réponse du poids sec des parties aériennes à la fumure azotée, et les traitements des autres essais sur lesquels la teneur en azote des parties aériennes était supérieure à 4 p. 100 (azote non limitant, d'après la figure 1).

L'examen des séquences de tallage herbacé permet de distinguer les essais sur lesquels il n'y a pas encore de compétition entre plantes au début de la montaison de ceux sur lesquels la compétition est déjà intervenue :

+ pour 20 p. 100 des essais (11 sur 56), les talles présentes au début de la montaison sont celles que laisse prévoir le modèle théorique d'émission des feuilles et des talles établi par MASLE $(1981 b)$,

+ pour les autres essais (45 sur 56), le tallage est, au début de la montaison, arrêté depuis plus d'un phyllochrone pour au moins 50 p. 100 des plantes. La nature des traitements considérés ici (azote non limitant) et les contrôles effectués sur le milieu (bilans hydriques, analyses de sol) incitent à attribuer cet arrêt du tallage à une compétition pour la lumière.

Pour le $1^{\text {er }}$ groupe d'essais, la biomasse aérienne est toujours inférieure à $70 \mathrm{~g} / \mathrm{m}^{2}$ et, pour le $2^{\mathrm{e}}$ groupe, toujours supérieure à $50 \mathrm{~g} / \mathrm{m}^{2}$ (tabl. 4). La biomasse aérienne seuil est donc comprise, pour les 3 variétés
TABLEAU 4

Biomasse aérienne par unité de surface au début de la montaison et arrêt du tallage (résultat de 56 essais sur les variétés "Talent", " Fidel 》, « Lutin »).

Aerial biomass per unit area at the beginning of the elongation phase and end of tillering.

\begin{tabular}{ccc}
\hline $\begin{array}{c}\text { Biomasse aérienne } \\
\left(\mathrm{g} / \mathrm{m}^{2}\right)\end{array}$ & $\begin{array}{c}\text { Arrêt du tallage } \\
\text { sur moins de } 50 \% \\
\text { des plantes à } \\
\text { l'observation }\end{array}$ & $\begin{array}{c}\text { Arrêt du tallage } \\
\text { sur plus de } 50 \% \\
\text { des plantes à } \\
\text { l'observation }\end{array}$ \\
\hline $\begin{array}{c}<50 \\
>\text { et } \leqslant 70\end{array}$ & 5 & 0 \\
$>70$ & 6 & 8 \\
\hline
\end{tabular}

étudiées, entre 50 et $70 \mathrm{~g} / \mathrm{m}^{2}$. Sur la base du rapport surface foliaire/biomasse, observé par MASLE (1985) pour nos variétés $\left(170 \mathrm{~cm}^{2} / \mathrm{g}\right)$, ce seuil correspond à un indice foliaire de 0,85 à 1,20 . Ces chiffres sont cohérents avec ceux déduits du suivi de croissance effectué par PUCKRIDGE \& DONALD (1967) sur 5 densités de peuplement échelonnées : la compétition pour la lumière n'est intervenue avant le début de la montaison que pour les 2 densités les plus fortes, dont la biomasse aérienne dépassait à cette époque $70 \mathrm{~g} / \mathrm{m}^{2}$ et l'indice foliaire 1,3 .

\section{Ajustement du modèle aux données de Champagne crayeuse et du Noyonnais}

Le modèle décrit par les relations (8) et (9) a été paramétré pour chaque région :

- par variété : «Talent» en Champagne, « Fidel » et « Lutin » dans le Noyonnais ;

- par gamme de date de semis, correspondant à des régimes thermiques différents. L'étude climatique des 2 régions a amené à distinguer 3 périodes :

- du 5 au 25/10 : normales pentadaires supérieures à $10^{\circ} \mathrm{C}$;

- du 26/10 au 10/11 : normales pentadaires comprises entre 7 et $8{ }^{\circ} \mathrm{C}$;

- du $11 / 11$ au $31 / 12$ : normales pentadaires inférieures $6^{\circ} \mathrm{C}$.

En 1979, où les semis d'octobre et de novembre ont été, en Champagne, très touchés par le gel (perte de 50 p. 100 ou plus des plantes), on a substitué à cette subdivision une distinction blés gelés-blés non gelés, levés en février après la période de froid intense (semis de décembre et de février) :

- le cas échéant, par type de sol, lorsque ses variations dans la petite région pouvaient induire des microclimats de la culture différents : cas, en Champagne crayeuse, des «terres blanches ", à réchauffement printanier lent, distinguées des «terres rouges", à réchauffement plus rapide (SEBILlotTE et al., 1978 ; BOIFFIN et al., 1982).

Le coefficient $\alpha$ de la relation (8) a été calculé en prenant en compte tous les essais où la biomasse aérienne $/ \mathrm{m}^{2}$ était inférieure à $70 \mathrm{~g} / \mathrm{m}^{2}$. Les coefficients $a$ et $b$ de la relation (9) ont été établis par ajustement linéaire de NP/BA 1 à NP, en prenant en compte tous les essais où la biomasse aérienne était 
TABLEAU 5

Résultats de l'ajustement du modèle de variation de BAIm en fonction de NP aux données de Champagne crayeuse et du Noyonnais. Fitting values of the relation between BAlm and number of plants (see text) (experimental data from Champagne crayeuse and Noyonnais).

\begin{tabular}{|c|c|c|c|c|c|c|c|c|c|c|c|c|}
\hline \multirow[b]{2}{*}{ Région } & \multirow[b]{2}{*}{ Variété } & \multirow[b]{2}{*}{ Période de semis } & \multicolumn{4}{|c|}{$\begin{array}{l}\text { Relation (8) : BAlm }=\alpha \mathrm{NP} \\
\quad \text { pour BA1m }<70 \mathrm{~g} / \mathrm{m}^{2}\end{array}$} & \multirow[b]{2}{*}{$\begin{array}{l}\text { Coefficient } \\
\text { de corréla- } \\
\text { tion entre } \\
\text { NP et } \frac{N P}{\text { BAlm }}\end{array}$} & \multicolumn{4}{|c|}{$\begin{array}{l}\text { Relation (9) : BA } 1 \mathrm{~m}=\frac{\mathrm{NP}}{\text { pour BA1m }>50 \mathrm{~g} / \mathrm{m}^{2}}=\frac{\mathrm{a}+\mathrm{bNP}}{}\end{array}$} & \multirow{2}{*}{$\begin{array}{c}\frac{1-\mathrm{b} \cdot \mathrm{BA} 1 \mathrm{~s}}{\mathrm{a}} \\
\text { (pour } \\
\text { BA } 1 \mathrm{~s}= \\
60 \mathrm{~g} / \mathrm{m}^{2} \\
\text { (g/plante) }\end{array}$} \\
\hline & & & $\begin{array}{c}\alpha \\
\text { calculé } \\
\text { (g/plante) }\end{array}$ & $\begin{array}{c}\text { Effectif } \\
\text { de } \\
\text { parcelles }\end{array}$ & $\begin{array}{c}\text { Gamme de } \\
\text { NP couverte } \\
\text { par les } \\
\text { parcelles }\end{array}$ & $\begin{array}{c}\text { Coefficient } \\
\text { de variation } \\
\text { de } \alpha \text { calculé } \\
\sigma_{0}\end{array}$ & & $\begin{array}{c}\mathrm{a} \\
\text { calculé } \\
\left(\mathrm{g}^{-1}\right)\end{array}$ & $\begin{array}{c}\mathrm{b} \\
\text { calculé } \\
\left(\mathrm{m}^{2} \cdot \mathrm{g}^{-1}\right)\end{array}$ & $\begin{array}{l}\text { Effectif } \\
\text { de } \\
\text { parcelles }\end{array}$ & $\begin{array}{c}\text { Gamme de } \\
\text { NP couverte } \\
\text { par les } \\
\text { parcelles }\end{array}$ & \\
\hline \multirow{5}{*}{$\begin{array}{l}\text { Champagne } \\
\text { crayeuse }\end{array}$} & $\mathrm{T}$ & $-11 / 11$ au $31 / 12$ & & & & & & & & & & \\
\hline & a & "Terres blanches" & 0,179 & 13 & 120500 & 14 & 0,62 & 3,48 & 0,00650 & 9 & 300580 & 0,175 \\
\hline & 1 & «Terres rouges» & 0,210 & 6 & 280330 & 8 & - & - & - & - & - & - \\
\hline & $\begin{array}{l}\mathrm{e} \\
\mathrm{n}\end{array}$ & $\begin{array}{l}\text { Déc. } 78 \text { et fév. } 79 \\
- \text { Oct. } / \text { Nov. } 78\end{array}$ & 0,141 & 7 & 150550 & 24 & 0,81 & 3,48 & 0,00927 & 4 & 290550 & 0,128 \\
\hline & $\mathrm{t}$ & Blés gelès & 0,073 & 7 & 50250 & 18 & - & - & - & - & - & - \\
\hline \multirow{3}{*}{ Noyonnais } & $\begin{array}{l}\mathrm{F} \\
\mathrm{i}\end{array}$ & $5 / 10$ au $25 / 10$ & 0,435 & 2 & 100160 & - & 0,97 & 1,44 & 0,00649 & 6 & 100290 & 0,426 \\
\hline & d & $26 / 10$ au $10 / 11$ & 0,324 & 9 & 140300 & 24 & 0,81 & 1,48 & 0,00818 & 15 & 150420 & 0,344 \\
\hline & 1 & $11 / 11$ au $31 / 12$ & 0,280 & 6 & 150300 & 11 & - & - & 一 & - & - & - \\
\hline \multirow{3}{*}{ Noyonnais } & $\begin{array}{l}\mathbf{L} \\
\mathrm{u}\end{array}$ & $5 / 10$ au $25 / 10$ & 0,407 & 3 & 130180 & 15 & 0,87 & 1.87 & 0,00379 & 9 & 130330 & 0,412 \\
\hline & $\mathrm{t}$ & $26 / 10$ au $10 / 11$ & 0,296 & 16 & 90240 & 16 & 0,78 & 1,64 & 0,00900 & 14 & 190360 & 0,260 \\
\hline & $i$ & $11 / 11$ аи $31 / 12$ & 0.197 & 4 & 210380 & 9 & 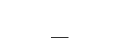 & 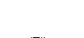 & 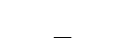 & & & - \\
\hline
\end{tabular}

supérieure à $50 \mathrm{~g} / \mathrm{m}^{2}$. Les résultats sont donnés dans le tableau 5.

Le modèle se révèle globalement satisfaisant :

- Pour chaque combinaison variété-lieu-période de semis, le poids sec d'une plante est peu variable, au-dessous de $70 \mathrm{~g}$ de biomasse aérienne $/ \mathrm{m}^{2}$ (coefficient de variation de $\alpha$ inférieur à 25 p. 100) ; au-delà de $50 \mathrm{~g}$ de biomasse aérienne $/ \mathrm{m}^{2}$, les variations du nombre de plantes $/ \mathrm{m}^{2}$ rendent compte de la majeure partie des variations de l'inverse du poids sec d'une plante $\left(r^{2} \geqslant 0,60\right.$, sauf pour un groupe d'essais). On vérifie enfin que, dans tous les cas, $\alpha$ est proche de $1-$ b.BA 1s ainsi que le laisse prévoir la relation $(10)$.

- L'écart maximum entre le modèle et les valeurs réelles est de $20 \mathrm{~g} / \mathrm{m}^{2}$; il ne dépasse $10 \mathrm{~g} / \mathrm{m}^{2}$ que dans 14 cas sur 84 . Ces erreurs restent faibles en regard de la grande variabilité de BA 1.

Nous ne disposons pas d'un nombre d'essais suffisant chaque année pour tester l'existence d'un effet « année » sur la valeur des paramètres. Cependant, la répartition aléatoire des essais des différentes années dans les histogrammes des résidus de régression linéaire autorise à considérer qu'il est limité. Dans la mesure où, entre les différentes années d'étude, nous avons exploré une partie importante de la gamme des températures d'hiver et de printemps possibles dans chaque région, le modèle proposé paraît utilisable à des fins prédictives.

\section{DISCUSSION-CONCLUSION}

\section{Comparaison des références issues du modèle avec les références antérieures}

A l'issue de l'analyse, les besoins du blé jusqu'au début de la montaison apparaissent très variables entre situations culturales. Dans nos conditions, ils varient pour un même pédoclimat et une même variété dans un rapport de 1 à 4, même en l'absence de gel hivernal ( 20 à $80 \mathrm{~kg} \mathrm{~N} / \mathrm{ha}$ environ). Etant donné l'incidence considérable du régime thermique hivernal sur la croissance, la variation des besoins selon le pédoclimat est elle-même certainement importante. La norme de $45 \mathrm{~kg} \mathrm{~N} / \mathrm{ha}$, proposée par RÉMY \& HÉBERT (1977) pour l'évaluation des besoins précoces, n'est donc $a$ priori utilisable que pour une situation moyenne dans le Bassin parisien, conditions dans lesquelles elle a été établie. La mise au point de références régionales, pour laquelle le modèle proposé pourrait servir de base, apparaît nécessaire.

D'après ce modèle, les besoins du blé augmentent avec la densité pour une même date de semis et sont, à même densité, significativement plus importants pour les semis précoces que pour les semis tardifs. Dans nos conditions, les besoins doublent approximativement quand la densité de plantes passe de 150 à 400 plantes $/ \mathrm{m}^{2}$ ou quand le semis est avancé du 25 novembre au 15 octobre. Ces variations sont en contradiction avec les grilles d'évaluation de l'apport " tallage " diffusées dans les 15 dernières années en France (par exemple RÉMY, 1974 ; RÉMY \& HÉBERT, 1977 ; I.T.C.F., 1978) qui proposent d'augmenter les doses d'engrais sur les blés clairs ou peu avancés en sortie d'hiver par l'intermédiaire d'un coefficient « $\mathrm{k} »$ variant de 1 à 3 :

$$
\text { Dose tallage }(\mathrm{kg} \mathrm{N} / \mathrm{ha})=(45-\mathrm{Q}) \mathrm{k}-\mathrm{A}(11)
$$

où $\mathrm{A}$ est la fourniture d'azote par le sol entre la sortie de l'hiver et le début de la montaison ; 45 représente les besoins du blé jusqu'au début de la montaison ; $\mathrm{Q}$, la quantité d'azote déjà absorbé par le blé en fin d'hiver.

La présence de ce coefficient « $\mathrm{k}$ » dans le bilan prévisionnel ne serait justifiée que si la fumure azotée avait un effet sur le développement de la culture, ce qui ne semble pas le cas (MASLE-MEYNARD, 1981a), ou si l'absorption de l'engrais était limitée sur les blés 
clairs ou semés tard par un enracinement peu dense ou peu profond au stade "épi $1 \mathrm{~cm}$ 》; les observations d'enracinement effectuées sur nos essais conduisent à écarter cette éventualité, sauf en cas de perte de pieds par déchaussement.

Cette contradiction entre nos conclusions et les conceptions classiques doit être rapprochée de l'évolution historique de l'époque de réalisation de l'apport «montaison ». Dans les expériences de CoÏC (1956), ou du S.P.I.E.A. (1968), le $2^{\mathrm{e}}$ apport était effectué dans le courant de la montaison (épi à $5 \mathrm{ou} 10 \mathrm{~cm}$ du plateau de tallage). Il n'avait donc qu'un rôle mineur sur la formation du nombre d'épis, qui dépendait surtout de l'apport " tallage ». Sur les blés clairs, où le nombre d'épis est souvent limitant pour le rendement, on tendait à augmenter la part de cet apport. On sait aujourd'hui que, pour obtenir un nombre d'épis élevé, il est préférable d'effectuer le $2^{\mathrm{e}}$ apport d'azote au stade «épi $1 \mathrm{~cm}$ » (VIAUX, 1980). Le rôle du $1^{\text {er }}$ apport s'en trouve réduit, en particulier sur un blé clair où il a peu d'effet sur la biomasse aérienne.
Le modèle proposé pour le calcul du $1^{\text {er }}$ apport d'engrais azoté est donc contingent, dans nos expérimentations, du stade de l'apport montaison. Les situations où celui-ci ne peut pas être effectué au stade " épi $1 \mathrm{~cm}$ » ou risque de ne pas être immédiatement utilisable seraient justiciables de références spécifiques.

Recu le 18 avril 1984. Accepté le 20 février 1985.

\section{REMERCIEMENTS}

Je remercie le CETA de Romilly (Aube) et l'ADANE (Oise), sans le concours desquels l'étude présentée ici n'aurait pu être réalisée, ainsi que les agriculteurs qui ont accueilli les essais sur leurs parcelles. Je tiens également à remercier mes collègues de la Chaire d'Agronomie de l'INA-PG de m'autoriser à utiliser des données acquises en commun en Champagne.

Une partie des expériences effectuées dans le Noyonnais, a été financée par l'action incitative du Ministère de l'Agriculture: « Reproductibilité des systèmes de production dans le Noyonnais ».

ANNEXE 1

Données climatiques.

Climatic data.

Pluviométrie mensuelle (mm)

\begin{tabular}{crrrrrrrrrr}
\hline \hline Station de Romilly-sur-Seine & $\mathrm{O}$ & $\mathrm{N}$ & $\mathrm{D}$ & $\mathrm{J}$ & $\mathrm{F}$ & $\mathrm{M}$ & $\mathrm{A}$ & $\mathrm{M}$ & $\mathrm{J}$ & $\mathrm{J}$ \\
\hline $1976-77$ & 79 & 53 & 53 & 56 & 100 & 55 & 33 & 69 & 83 & 65 \\
$1977-78$ & 54 & 81 & 44 & 76 & 58 & 156 & 49 & 67 & 62 & 45 \\
$1978-79$ & 9 & 14 & 110 & 37 & 78 & 104 & 61 & 64 & 59 & 24 \\
$1979-80$ & 90 & 66 & 96 & 44 & 61 & 65 & 14 & 43 & 75 & 82 \\
\hline Station de Saint-Quentin & & & & & & & & & \\
\hline $1980-81$ & 85 & 56 & 69 & 92 & 33 & 70 & 12 & 95 & 78 & 82 \\
$1981-82$ & 166 & 38 & 100 & 59 & 14 & 53 & 32 & 108 & 110 & 15 \\
$1982-83$ & 77 & 69 & 114 & 59 & 54 & 59 & 58 & 85 & 66 & 37 \\
\hline
\end{tabular}

Température moyenne mensuelle $\left({ }^{\circ} \mathrm{C}\right)$

\begin{tabular}{ccccccccccc}
\hline \hline Station de Romilly-sur-Seine & $\mathrm{O}$ & $\mathrm{N}$ & $\mathrm{D}$ & $\mathrm{J}$ & $\mathrm{F}$ & $\mathrm{M}$ & $\mathrm{A}$ & $\mathrm{M}$ & $\mathrm{J}$ & $\mathrm{J}$ \\
\hline $1976-77$ & 12,3 & 6,3 & 1,7 & 3,6 & 6,6 & 8,1 & 8,1 & 12,8 & 15,5 & 17,4 \\
$1977-78$ & 12,6 & 7,0 & 4,9 & 2,6 & 2,9 & 7,2 & 7,8 & 12,5 & 15,0 & 16,6 \\
$1978-79$ & 10,4 & 4,7 & 4,8 & $-1,7$ & 3,2 & 6,8 & 8,6 & 12,3 & 16,4 & 17,6 \\
$1979-80$ & 11,8 & 5,9 & 5,7 & 1,0 & 6,0 & 8,2 & 8,1 & 11,7 & 15,0 & 16,3 \\
\hline Station de Saint-Quentin & & & & & & & & & & \\
\hline $1980-81$ & 9,2 & 4,8 & 3,2 & 3,0 & 2,4 & 9,3 & 9,2 & 12,5 & 14,6 & 16,6 \\
$1981-82$ & 9,5 & 6,5 & 2,3 & 2,7 & 3,6 & 5,7 & 8,0 & 12,6 & 16,8 & 18,4 \\
$1982-83$ & 10,3 & 7,0 & 3,7 & 5,6 & 1,5 & 6,2 & 8,6 & 10,8 & 16,6 & 21,0 \\
\hline \hline
\end{tabular}


ANNEXE 2

Caractéristiques des sols des essais complets.

Main characteristics of the soils of the complete trials.

\begin{tabular}{|c|c|c|c|c|c|}
\hline & Essai & $\mathrm{CaCO}_{3}$ total & $\begin{array}{c}\text { Analyse de la couche arable } \\
\text { Teneur en } \% \text { de terre fine } \\
\text { Argile }\end{array}$ & Matière organique & $\begin{array}{c}\text { Profondeur } \\
\text { d'enracinement } \\
\text { à la floraison } \\
\text { (cm) (1) }\end{array}$ \\
\hline 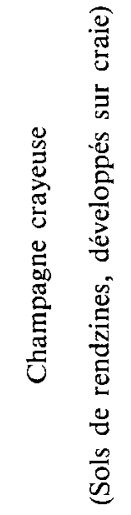 & $\begin{array}{c}\text { EER } \\
\text { EEB } \\
\text { EON } \\
\text { ED2 } \\
\text { EN1 } \\
\text { EN2 } \\
\text { EN3-1,2,3 } \\
\text { ES2-I,M } \\
\text { ED5-A,D } \\
\text { EN5 } \\
\text { ES1-I,K } \\
\text { ED6A } \\
\text { EN6 }\end{array}$ & $\begin{array}{l}66 \\
72 \\
78 \\
73 \\
79 \\
54 \\
77 \\
68 \\
80 \\
79 \\
71 \\
76 \\
75\end{array}$ & $\begin{array}{l}- \\
- \\
- \\
- \\
- \\
- \\
- \\
- \\
- \\
-\end{array}$ & $\begin{array}{l}2,7 \\
2,8 \\
3,9 \\
3,0 \\
3,0 \\
2,6 \\
3,5 \\
3,9 \\
2,8 \\
3,6 \\
3,6 \\
2,9 \\
2,5\end{array}$ & $\begin{array}{l}60 \\
55 \\
55 \\
90 \\
90 \\
60 \\
50 \\
70 \\
70 \\
80 \\
40 \\
40 \\
60\end{array}$ \\
\hline 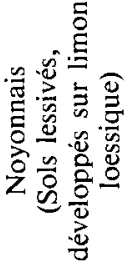 & $\begin{array}{c}\text { AZ2 } \\
\text { FA2 } \\
\text { I5-A,B } \\
\text { FA5 } \\
\text { FA6 } \\
\text { FA9 } \\
\text { FA1 }\end{array}$ & $\begin{array}{l}0 \\
2 \\
0 \\
0 \\
0 \\
1 \\
0\end{array}$ & $\begin{array}{l}17,4 \\
18,5 \\
16,6 \\
20,3 \\
14,9 \\
18,1 \\
19,1\end{array}$ & $\begin{array}{l}1,8 \\
1,4 \\
1,3 \\
1,3 \\
1,3 \\
1,5 \\
1,4\end{array}$ & $\begin{array}{r}110 \\
130 \\
70 \\
80 \\
80 \\
120 \\
120\end{array}$ \\
\hline
\end{tabular}

(1) Profondeur en dessous de laquelle la densité de racines est inférieure à une racine/2 $\mathrm{dm}^{2}$ de face verticale du profil.

\section{RÉFÉRENCES BIBLIOGRAPHIQUES}

Aase J. K., 1978. Relationship between leaf area and dry matter in winter wheat. Agron. J., 70, 563-564.

Baldy C., 1973. Progrès récents concernant l'étude du système racinaire du blé (Triticum sp.). Ann. Agron., 24 (2), 241-276.

Boiffin J., Caneill J., Meynard J. M., Sebillotte M., 1981. Elaboration du rendement et fertilisation azotée du blé d'hiver. I. - Protocole et méthode d'étude d'un problème technique régional. Agronomie, 1 (7), 547-558.

Boiffin J., Meynard J. M., Sebillotte M., Caneill J., 1982. - Elaboration du rendement et fertilisation azotée du blé d'hiver. III. Influence des variations pédologiques ; conséquences pour la conduite de la culture. Agronomie, 2 (5), 417-428.

Chatilov I. S., Safonov A. F., 1972. Les teneurs en azote, phosphore et potassium des racines de blé d'hiver. Izv. Timiryazevsk. s-kh. Akad., (4), 223-226 (en russe).

Coïc Y., 1949. L'apport, à dose normale et à une époque favorable, d'engrais azotés immédiatement assimilables peut provoquer ultérieurement un besoin supplémentaire d'azote. C.R. Acad. Agric. Fr., 35 (2), 79-82.

Coïc Y., 1956. La nutrition et la fertilisation du blé d'hiver. Les besoins en azote du blé d'hiver. Conséquences agronomiques. Ann. Agron., 7 (1), 115-131.

Donald C. M., 1963. Competition among crop and pasture plants. Adv. Agron., 15, 1-118.

Hébert J., 1969. La fumure azotée du blé d'hiver. Bull. Tech. Inform. Min. Agric., 244, 755-766.

Hodges T., Kanemasu E. T., 1977. Modeling daily dry matter production of winter wheat. Agron. J., 69, 974-978.

I.T.C.F., 1978. La fertilisation azotée du blé, 30 p.

Jonard P., 1964. Etude comparative de la croissance de deux variétés de blé tendre. Ann. Amélior. Plantes, 14 (2), 101-130.
Jonard P., Odent M., 1967. Etude de l'évolution de l'azote au cours de la croissance de la tige principale du blé tendre. Ann. Amélior. Plantes, 17 (1), 23-31.

Karlen D. L., Whitney D. A., 1980. Dry matter accumulation, mineral concentrations and nutrient distribution in winter wheat. Agron. J., 72 (2), 281-288.

Keulen (Van) M., de Milliano W. A. J., 1984. Potential wheat yields in Zambia. A simulation approach. Agricultural systems, 14, 171-192.

Masle J., 1985. Analyse des relations de compétition pour la lumière dans un peuplement de blé d'hiver. A paraître in Agronomie, 5.

Masle-Meynard J., 1980. L'élaboration du nombre d'épis chez le blé d'hiver. Influence de différentes caractéristiques de la structure du peuplement sur l'utilisation de l'azote et de la lumière. Thèse INA-PG, Paris, 201 p. + annexes.

Masle-Meynard J., 1981a. Relations entre croissance et développement pendant la montaison d'un peuplement de blé d'hiver. Influence des conditions de nutrition. Agronomie, 1 (5), 365-374.

Masle-Meynard J., 1981b. Elaboration du nombre d'épis d'un peuplement de blé d'hiver en situation de compétition pour l'azote. I. - Mise en évidence d'un stade critique par rapport à la montée d'une talle. Agronomie, 1 (8), 623-632.

Meynard J. M., 1983. Perspectives pour la conduite des blés clairs. C.R. Acad. Agric. Fr., 69 (11), 830-838.

Meynard J. M., Boiffin J., Caneill J., Sebillotte M., 1981. Elaboration du rendement et fertilisation azotée du blé d'hiver. II. - Types de réponse à la fumure azotée et application de la méthode du bilan prévisionnel. Agronomie, 7 (9), 795-806.

Meynard J. M., Boiffin J., Sebillotte M., 1982. Prediction of nitrogen fertilizer for winter wheat. Test of model. Proc. 9th Int. Plant Nutrition Coll. Ed. by A. Scaife, London, C.A.B., 390-395. 
Meynard J. M., Sebillotte M., 1982. Diagnostic sur les causes de variations du rendement du blé dans une petite région. La fatigue des sols, $23^{e}$ Coll. SFP, éd. I.N.R.A. Publ. (Les Colloques de l'I.N.R.A. ${ }^{\circ}$ 17), 157-168.

Puckridge D. W., Donald C. M., 1967. Competition among wheat plants sown at a wide range of densities. Aust. J. Agric. Res., 18, 193-211.

Puia I., Marcu T., Kain I., 1978. Some peculiarities of N, P, K proportion and contents during ontogenesis in winter wheat. Inst agron. « Dr Petru Groza » (Cluj-Napoca, Roum.) - Nodulae Botanicae horti agrobotanici (1977-78), IX, 109-113.

Rémy J. C., 1974. Pas de recettes passe-partout pour la fertilisation du blé. Fermes modernes, $\mathrm{n}^{\circ}$ spécial "Le blé ; céréale d'avenir", 89-96.

Rémy J. C., 1981. Etat actuel et perspective de la mise en œuvre des techniques de prévision de la fumure azotée. C.R. Acad. Agric. Fr., $67(10), 859-872$

Rémy J. C., Hébert J., 1977. Le devenir des engrais azotés dans le sol. C.R. Acad. Agric. Fr., 63 (11), 700-714.

Rémy J. C., Viaux P., 1980. Evolution des engrais azotés dans le sol. Perspectives Agricoles, 43, 5-9.
Rickman R. W., Ramig R. E., Allmaras R. R., 1975. Modeling dry matter accumulation in dryland winter wheat. Agron. J., 67 (3), 283-289.

Saut M. H., 1981. Contribution à l'étude des relations sociales entre le blé d'hiver et le ray grass raide : rôle des caractéristiques respectives de développement et de croissance des deux espèces. Thèse INA$\mathrm{PG}, 222 \mathrm{p}$.

Sebillotte M., Boiffin J., Caneill J., Meynard J. M., 1978. Sécheresse et fertilisation azotée du blé d'hiver. Essai d'analyse de situations au champ par l'étude des composantes du rendement. Bull. Assoc. fr. Etud. Sol, (3), 197-214.

S.P.I.E.A., 1968. 589 essais de fumure azotée (céréales 1967), $170 \mathrm{p}$.

Viaux P., 1980. Fumure azotée des céréales d'hiver. Perspectives Agricoles, 43, 10-26.

Viaux P., 1983. Azote au tallage en 10 figures. Cultivar, 167, 68-70.

Willey R. W., Heath S. B., 1969. The quantitative relationships between plant population and crop yield. Adv. Agron., 21, 281-231.

Wit (de) C. T., Brouwer R., Penning de Vries F. W. T., 1971. A dynamic model of plant and crop growth. In Wareing P. F. \& Cooper J. P. «Potential crop production », Heinemann Educational Books, London, 117-142. 